\title{
]jfis
}

\section{Experimental Validation of Longitudinal Control of a Platoon of Vessels Established via the V-REP Simulator}

Myoung-Sung Kim ${ }^{1}$, Kwang-Kook Lee ${ }^{2}$, and Dong Hun $\mathrm{Kim}^{3}$

${ }^{1}$ Department of Mechatronics Engineering, Kyungnam University, Changwon, Korea

${ }^{2}$ Department of Naval Architecture \& Ocean System Engineering, Kyungnam University, Changwon, Korea

${ }^{3}$ Department of Electrical Engineering, Kyungnam University, Changwon, Korea
Received: Oct. 29, 2019

Revised : Nov. 30, 2019

Accepted: Dec. 3, 2019

Correspondence to: Dong Hun Kim (dhkim@kyungnam.ac.kr)

(CThe Korean Institute of Intelligent Systems

cCThis is an Open Access article distributed under the terms of the Creative Commons Attribution Non-Commercial License (http://creativecommons.org/licenses/ by-nc/3.0/] which permits unrestricted noncommercial use, distribution, and reproduction in any medium, provided the original work is properly cited.

\begin{abstract}
This study experimentally validates longitudinal control of a platoon of vessels established via the virtual robot experimentation platform (V-REP) simulator. For inter-vessel platooning, vessel positions are verified through GPS and the azimuth between vessels. A safety distance between the preceding and trailing vessels moving at variable speeds is proposed. The proposed method enables all the vessels to move, maintaining a compact formation, even when another vessel crosses the platoon of vessels because preceding vessels wait for trailing vessels. Results of extensive V-REP-based simulations indicate that the proposed approach is viable and effective in establishing longitudinal control of a platoon of vessels.
\end{abstract}

Keywords: Platooning, Vessel, Following, V-REP

\section{Introduction}

Lately, a variety of unmanned surface vessels are being developed globally, having numerous applications and progressively demonstrating increased industrial and military use. For autonomous unmanned surface vessel propulsion and navigation, accurate positioning and obstacle avoidance data is required.

Currently, as the economy progresses, total manned small vessel volume is continuously declining due to relatively high operating costs. These problems have led to a steady expansion of autonomous navigation study [1.2]. Although automation technologies in the United States, Germany, and Japan have led the way until now, many other countries such as China, Israel, and South Korea are also investing in technology development. Based on these technical skills, research related to track keeping control [3]-5], dynamic ship position [6]-8], and automatic movement control [9] is underway; with application research also proceeding for actual vessels.

Meanwhile, to reduce land-based labor costs, platoon technology was developed for maintaining the distance between a platoon of vehicles. A representative example is the recent development of autonomous driving trucks in The Netherlands, where two or three trucks connected by Wi-Fi are driven at a constant distance. Additionally, numerous platooning systems based on vehicle-to-vehicle (V2V) communication have been studied [10, 11].

Similarly, to reduce the labor costs at sea, related studies on platooning have been conducted [12]; land-based studies have been conducted extensively; however, considerable sea-based 
research is yet to be conducted. Although vehicle platooning has been studied, no way of maintaining the vessel spacing at sea has been specified. Therefore, this study introduces vessel platooning.

This study experimentally validates the longitudinal control of a platoon of vessels established via the virtual robot experimentation platform (V-REP) [13, 14]. Presently, there are several robot branch simulators both at home and abroad; for example, Gazebo, We-bots, V-REP, etc. Among these, V-REP, as an open-source application also free to researchers, is one of the most widely used simulators. In the platooning framework, the safety distance between a preceding and a trailing vessel moving at a variable speed is proposed. This method enables all vessels to move, maintaining a compact formation, even when another vessel crosses their track because the preceding vessel waits for the trailing vessel. There is no intention to tackle all possible navigational problems related to the longitudinal control of a platoon of vessels.

The remainder of the paper is organized as follows. Section 2 shows vessel composition in the V-REP environment. Section 3 explains a vessel model and controller design. In Section 4, the vessel platooning using preceding vessel variable safe distance and speed control is proposed. The V-REP simulation results are presented and discussed in Section 5. Finally, the paper is concluded in Section 6.

\section{Composition in V-REP Environment}

Here, a platoon of unmanned vessels is designed through V-REP as shown in Figure 1. Unmanned vessels are equally equipped with GPS [15] and LiDAR [16, 17] sensors because they must always be aware of their current and final coordinates to reach the intended destination. To this end, vessels are equipped with these sensors for avoiding obstacles during autonomous driving. Figure 2 shows a platoon of vessels.

\section{Vessel Model and Controller Design}

An environmental unmanned vessel kinematic formula as shown in Figure 2 is expressed below in Eq. (1) [18, 19],

$$
\begin{aligned}
\Delta x_{p i} & =\frac{\cos \theta_{p i} \times\left(v_{L i}+v_{R i}\right)}{2}=\cos \theta_{p i} \cdot v_{i}, \\
\Delta y_{p i} & =\frac{\sin \theta_{p i} \times\left(v_{L i}+v_{R i}\right)}{2}=\sin \theta_{p i} \cdot v_{i}, \\
\Delta \theta_{p i} & =\frac{v_{L i}+v_{R i}}{2}=w_{i},
\end{aligned}
$$

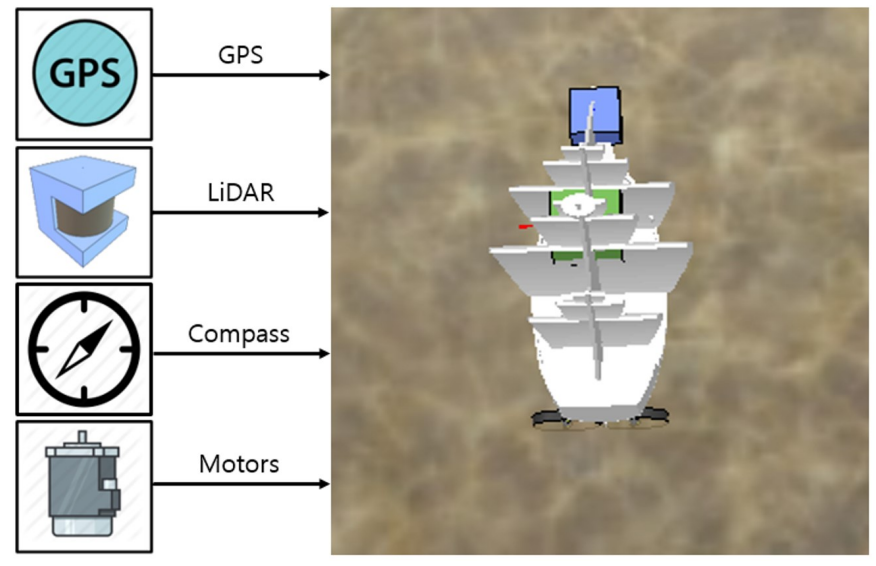

Figure 1. Vessel configuration in V-ERP.

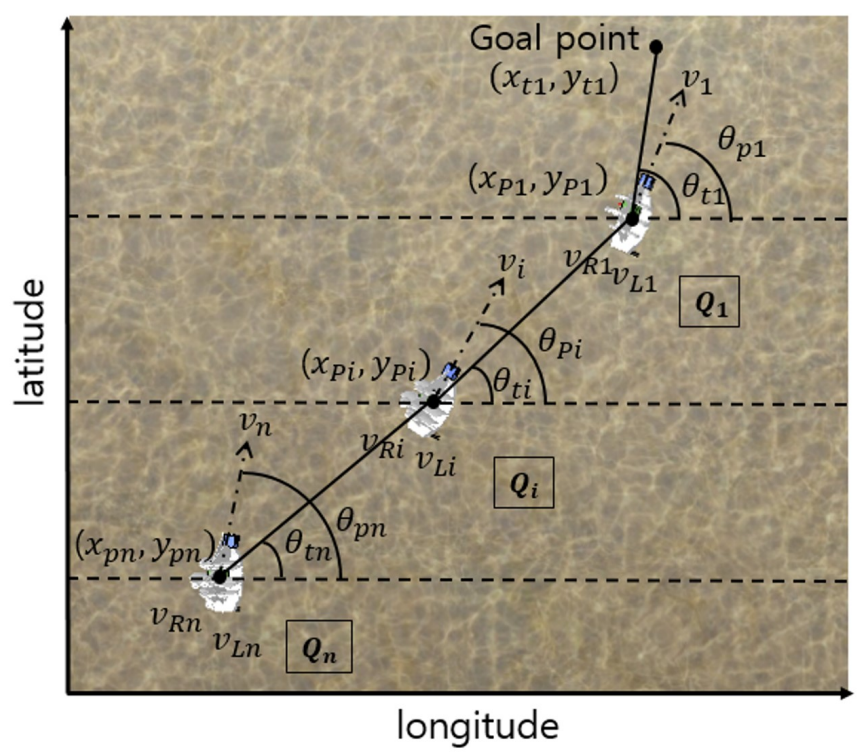

Figure 2. A platoon of vessels.

where $\theta_{p i}$ is the unmanned vessel rotation angle with respect to the $x$-axis. $v_{i}$ and $w_{i}$ is linear and angular velocity, respectively, and are defined by Eq. (5). $v_{L i}$ and $v_{R i}$ is the left and right propeller speed value, respectively.

Both propeller speeds of $v_{L i}$ and $v_{R i}$ are equal when driving straight. However, $v_{L i}$ is greater than $v_{R i}$ when the vessel turns right, and $v_{R i}$ is zero during a sharp right turn. The opposite is true when the vessel turns left.

Eq. (1) summarization is expressed in Eq. (2),

$$
\dot{Q}_{i}=\left[\begin{array}{c}
\dot{x}_{p i} \\
\dot{y}_{p i} \\
\dot{\theta}_{p i}
\end{array}\right]=\left[\begin{array}{cc}
\cos \theta_{p i} & 0 \\
\sin \theta_{p i} & 0 \\
0 & 1
\end{array}\right]\left[\begin{array}{c}
v_{i} \\
w_{i}
\end{array}\right] .
$$

The unmanned vessel has three degrees of freedom, $x_{p i}, y_{p i}$, 


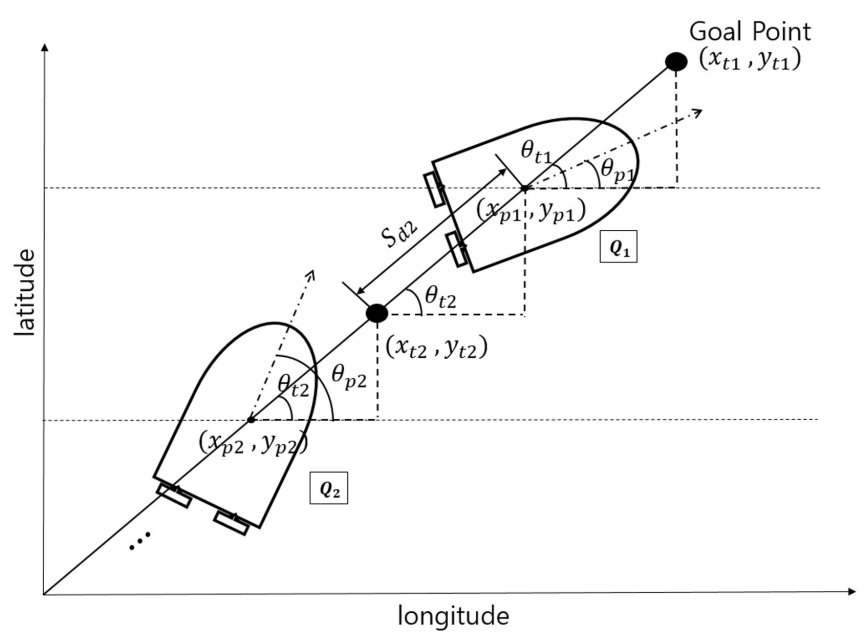

Figure 3. Safety distance between 1st vessel and 2nd vessel.

and $\theta_{p i}$, around the $z$-axis and is expressed as three vectors $Q_{i}=\left[\begin{array}{lll}x_{p i} & y_{p i} & \theta_{p i}\end{array}\right]^{T}$, where $x$ and $y$ are the $x$ - and $y$-axis positions.

The linear velocity $v_{i}$ and angular velocity $w_{i}$ are described in discrete time as follows:

$$
\begin{aligned}
v_{i}(k) & =\frac{v_{L i}(k)+v_{R i}(k)}{2}, \\
w_{i}(k) & =\frac{v_{L i}(k)-v_{R i}(k)}{2} .
\end{aligned}
$$

In Figure 3, $\left(x_{t 1}(k), y_{t 1}(k)\right)$ is the first vessel goal position. $\left(x_{t i}(k), y_{t i}(k)\right), i=2, \ldots, n$ is a vessel $Q_{i}$ goal position, which is designed according to $S_{d i}$,

$$
\begin{aligned}
& x_{t i}(k)=x_{p i-1}(k)-S_{d i}(k) \cdot \cos \left(\theta_{t i}(k),\right. \\
& y_{t i}(k)=y_{p i-1}(k)-S_{d i}(k) \cdot \sin \left(\theta_{t i}(k)\right),
\end{aligned}
$$

where compares the corresponding vessel starting point coordinates with the goal point coordinates. For a vessel $Q_{i}$, the current coordinates are $x_{p i}(k)$ and $y_{p i}(k)$. Figure 3 shows safety distance between 1st and 2nd vessels.

The vessel and goal point coordinate difference is $x_{d i}$ and $y_{d i}$ as follows:

$$
\begin{aligned}
& x_{d i}(k)=x_{t i}(k)-x_{p i}(k), \\
& y_{d i}(k)=y_{t i}(k)-y_{p i}(k) .
\end{aligned}
$$

The destination distance, $e_{d i}(k)$ is shown in Eq. (6),

$$
e_{d i}(k)=\sqrt{x_{d i}^{2}(k)+y_{d i}^{2}(k)} .
$$

The direction angle for the vessel goal position is $\theta_{t i}(k)$,

$$
\theta_{t i}(k)=\operatorname{atan}\left(\frac{y_{d i}(k)}{x_{d i}(k)}\right) .
$$

The vessel direction angle and goal position difference is,

$$
\theta_{d i}(k)=\theta_{t i}(k)-\theta_{p i}(k),
$$

where the $i$-th vessel direction angle is $\theta_{p i}(k)$.

The unmanned vessel control performs autonomous operation by comparing the current coordinates with the destination coordinates through the GPS and compass sensors. PD control is used because only azimuth direction and straight motion control are required [20, 21]. PD control is a control technique that uses derivative control to generate an error signal that produces a proportional control signal.

For a vessel $Q_{i}$, the left and right motor speed to a vessel goal point is as follows:

$$
\begin{aligned}
v_{L i}(k)= & c-k_{p}^{\theta} \theta_{d i}(k)-k_{d}^{\theta} \times \frac{\theta_{d i}(k)-\theta_{d i}(k-1)}{\Delta t} \\
& +k_{p}^{e} e_{d i}(k)+k_{d}^{e} \times \frac{e_{d i}(k)-e_{d i}(k-1)}{\Delta t}, \\
v_{R i}(k)= & c+k_{p}^{\theta} \theta_{d i}(k)+k_{d}^{\theta} \times \frac{\theta_{d i}(k)-\theta_{d i}(k-1)}{\Delta t} \\
& +k_{p}^{e} e_{d i}(k)+k_{d}^{e} \times \frac{e_{d i}(k)-e_{d i}(k-1)}{\Delta t},
\end{aligned}
$$

where $k_{p}^{\theta}$ and $k_{d}^{\theta}$ represent the proportional and differential gains for the direction angle, respectively; with $k_{p}^{e}$ and $k_{d}^{e}$ representing distance proportional and differential gains, respectively. $c$ is the minimum value required for the motor to turn.

The size of $v_{L}$ and $v_{R}$ is constrained using a saturation function due to vessel rotational radius.

The proportional terms use current steps of $\theta_{d i}(k)$ and $e_{d i}(k)$. The differential terms use variations of $\theta_{d i}(k)$ and $e_{d i}(k)$ for sampling time $\Delta \mathrm{t}$.

\section{Vessel Platooning}

To enable autonomous vessel platooning, a trailing vessel must secure a safe distance from its preceding vessel. The preferred safety distance must be as small as possible. The minimum safety distance can be defined in different forms when considering safety factors for tides, waves, or other external environmental factors.

The $i$-th unmanned vessel preferred safety distance rule is 
known as the California rule in Eq. (10) as follows [22]:

$$
S_{d i}(k)=\rho v_{i}(k)+L, \quad i=2, \ldots, n,
$$

where $v_{i}$ is the $i$-th vessel speed, $\rho$ is the minimum deceleration of the vessels and $L$ is the minimum distance, considerably larger than the vessel's length.

Based on the California rule, this study proposes a method that restricts the speed of the preceding vessel when its trailing vessel stops or encounters another vessel. According to Eq. (10), the vessel destination point is given by Eq. (4).

Eq. (10) is called a constant time headway policy for a certain time period; utilized for safety distance maintenance between preceding and trailing vessels at varying speeds. It also maintains a minimum distance of $L$ when the preceding vessel stops. When the constant $\rho$ is large, a significant preceding vessel speed percentage is used to establish safety distance rules. $S_{d 1}(k)$ is not needed, since the first vessel is going for the goal position. The vessel speed is expressed as the unit $1 \mathrm{kn}$ $=0.514 \mathrm{~m} / \mathrm{s}$.

Figure 4 shows an example of safety distance between the preceding and trailing vessels according to the preceding-ship speed when $\rho=0.5$ and $L=1.3 \mathrm{~m}$ in Eq. (10).

Eq. (11) is the compactness index and also represents the total safe distance between vessels. If the distance between vessels is small, a compact straight formation exists,

$$
\sum_{i=1}^{n-1} \sqrt{\left(x_{p i}-x_{p i+1}\right)^{2}+\left(y_{p i}-y_{p i+1}\right)^{2}} .
$$

Maintaining a safe distance between the preceding and trailing vessels means that the faster the preceding vessel speed, the larger the safety distance, and the slower the preceding vessel speed, the smaller the safety distance.

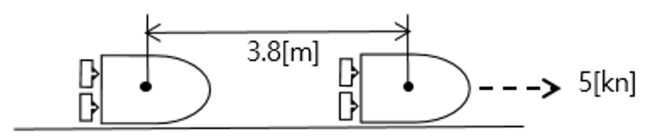

(a)

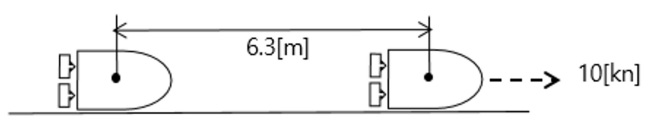

(b)

Figure 4. Example of safety distance according to speed: (a) $5 \mathrm{kn}$ and (b) $10 \mathrm{kn}$.
However, if the trailing vessel encounters another vessel interrupting its track, it stops until the interrupting vessel is away from its path. In this case, the distance between the trailing and preceding vessels increases further; as a result, the trailing vessel takes a long time to return to the preceding-vessel minimum safety distance.

To prevent this phenomenon, when the trailing vessel stops or reduces speed, we consider varying Gaussian speed as in Figure 5(a) for the preceding vessel, as shown in Eq. [12,

$$
v_{i}(k)=\exp \left(-\frac{\left(e_{d i}(k)-\mu\right)^{2}}{\sigma^{2}}\right),
$$

where $\sigma$ means the width and $\mu$ means the time to reach the maximum value.

Finally, we adopt a variable speed as in Figure 4(b) for the

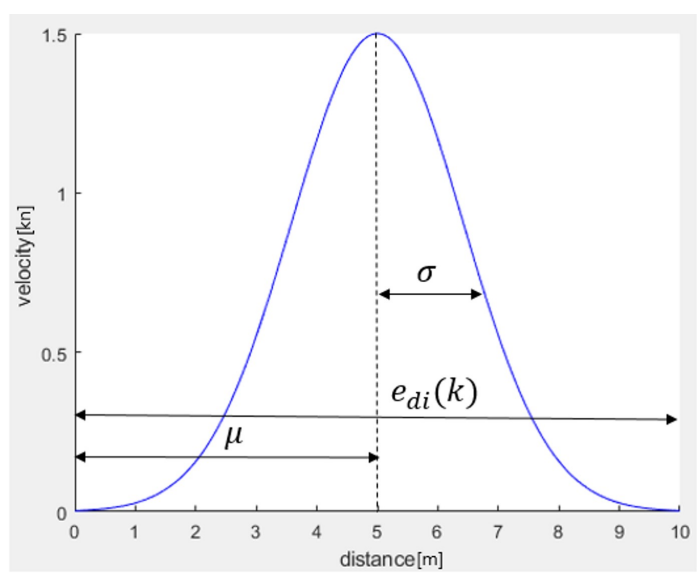

(a)

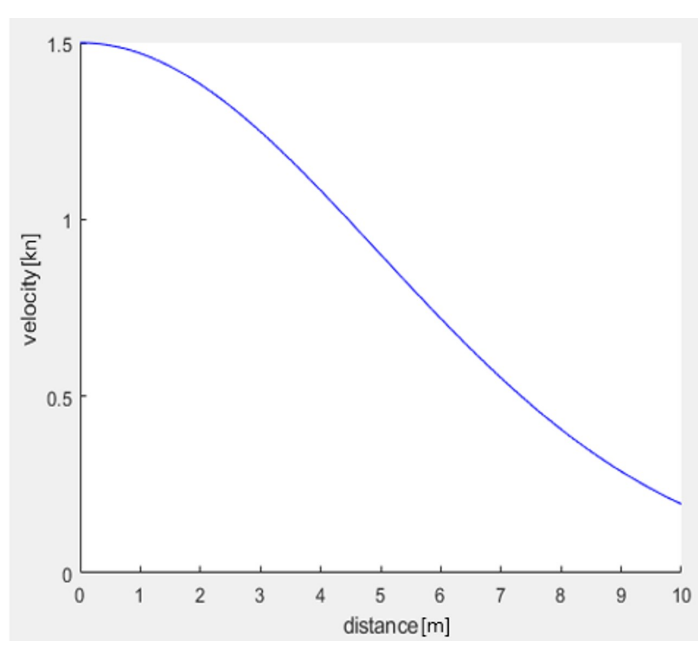

(b)

Figure 5. Speed of the preceding vessel in Eq. (12): (a) $\mu=5$ and $\sigma=2$ and (b) $\mu=0$ and $\sigma=7$. 
preceding vessel, where $\mu=0, \sigma=7$.

This paper presents experimental validation for platoon of vessels longitudinal control. Latitudinal control issues related to Collision regulations (COLREGs) is out of scope for the paper [23, 24]. If the trailing vessel encounters another vessel that interrupts the track, it stops until the interrupting vessel is out of the pathway; then, the trailing vessel follows the preceding vessel without changing direction. Thus, only a COLREGs crossing maneuver from right or left is considered.

\section{Simulation}

In this section, platoon of vessels longitudinal control is presented. The proposed method is demonstrated for three unmanned vessels through a V-REP simulator [9, 10]. The parameter values are $\rho=0.5, L=1.3 \mathrm{~m}, k_{p}^{\theta}=0.01, k_{d}^{\theta}=0.037$, $k_{p}^{e}=1$, and $k_{d}^{e}=0.1$, equally applied to three unmanned vessels.

Figures 6 and 7 show the V-REP simulation snap shots and vessels position coordinates, respectively. At 52 seconds, another vessel crosses between the first and second vessels. The second vessel waits while the intervening vessel passes the pla-

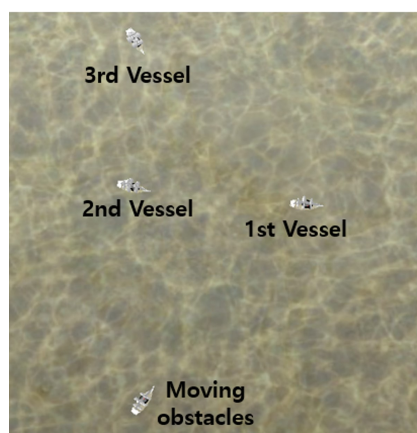

(a)

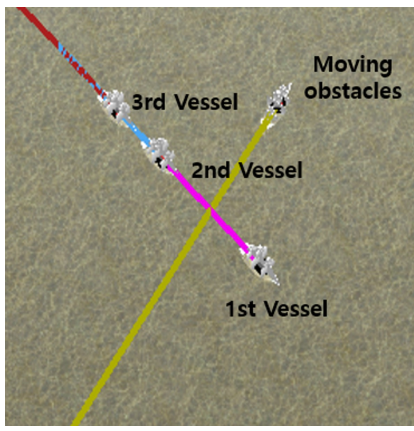

(c)

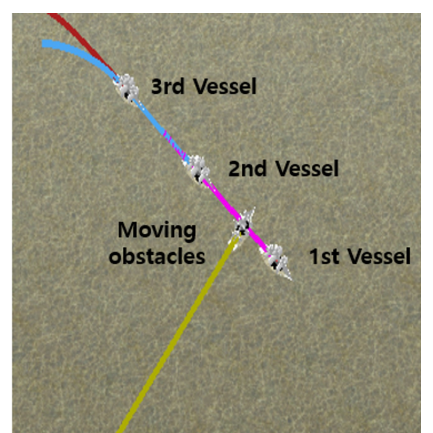

(b)

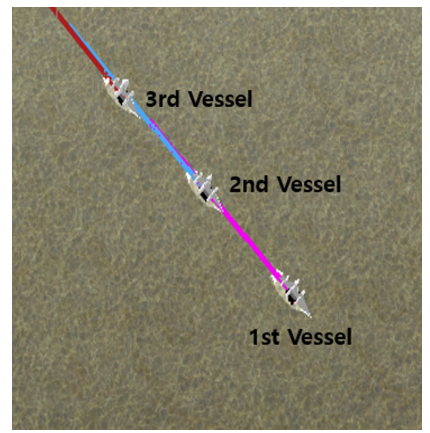

(d)
Figure 6. V-REP simulation: (a) $t=0$, (b) $t=52$, (c) $t=62$, and (d) $t=170$ seconds.

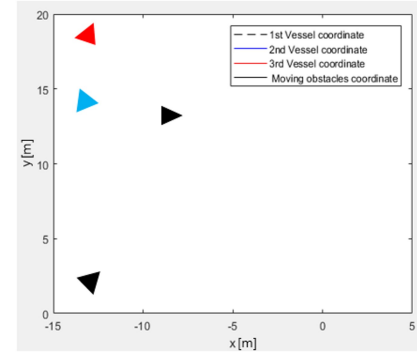

(a)

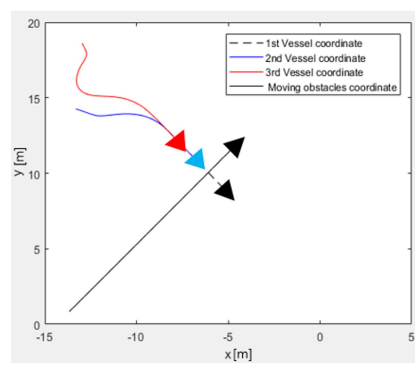

(c)

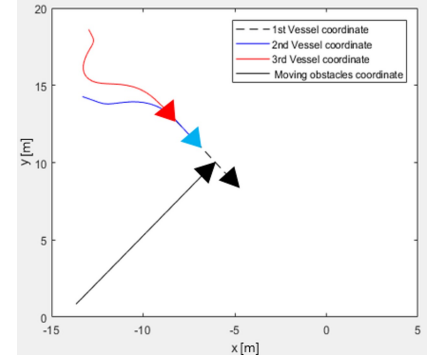

(b)

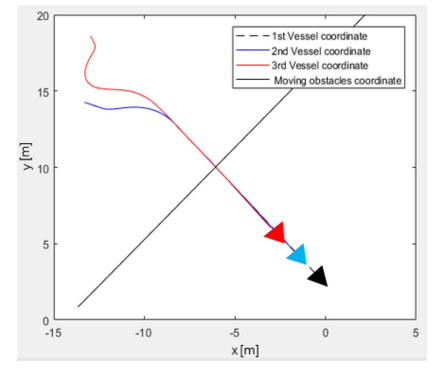

(d)
Figure 7. Position coordinates of vessels: (a) $t=0$, (b) $t=52$, (c) $t=62$, and (d) $t=170$ seconds.

toon. The first vessel waits for the second vessel, maintaining the gap between the two vessels.

For the scenario, linear velocity, angular velocity, and safety distance between the vessels are illustrated in Figures 8 and 9; showing a platoon of vessels based on a constant safety distance method and the proposed method, respectively. In Figure 9(a) the first vessel is waiting for the second vessel between 55 seconds and 70 seconds unlike Figure 8(a).

In the proposed method, safety distance changes linearly based on preceding vessel speed. At 75 seconds in Figure 9(c), the actual distance becomes smaller than the safety distance. This is caused by the drastic preceding vessel speed change; however, since the actual distance is still larger than minimum distance $L$, no collision occurs.

Figure 10 shows the total safe distance between vessels described in Equation (11). The constant safety distance method and the proposed method are used for vessel platooning in Figure 10(a) and 10(b), respectively. As a result, the proposed method shown in Figure 10(b) shows a more compact formation than the general method with constant safety distance defined by $L=2 \mathrm{~m}$ as seen in Figure 10(a). Therefore, the proposed method shows it is better suited for maintaining distance compactness between vessels. 


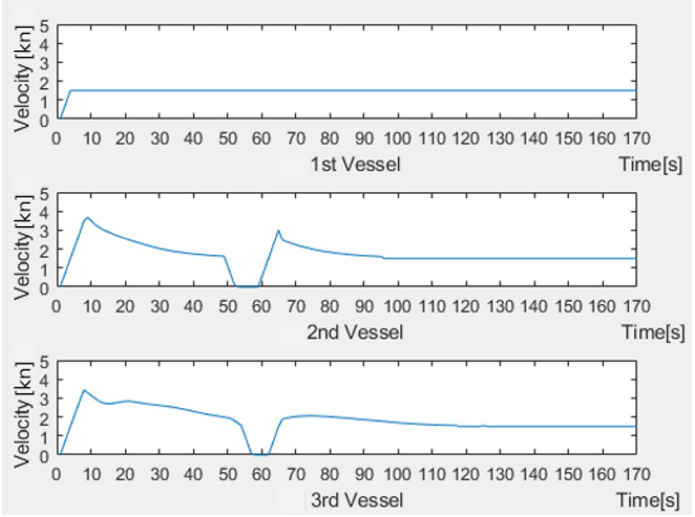

(a)
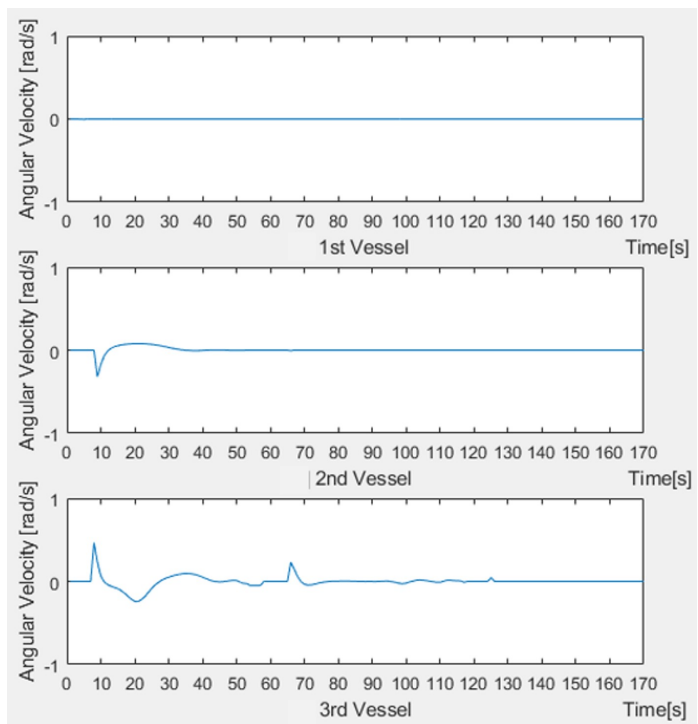

(b)

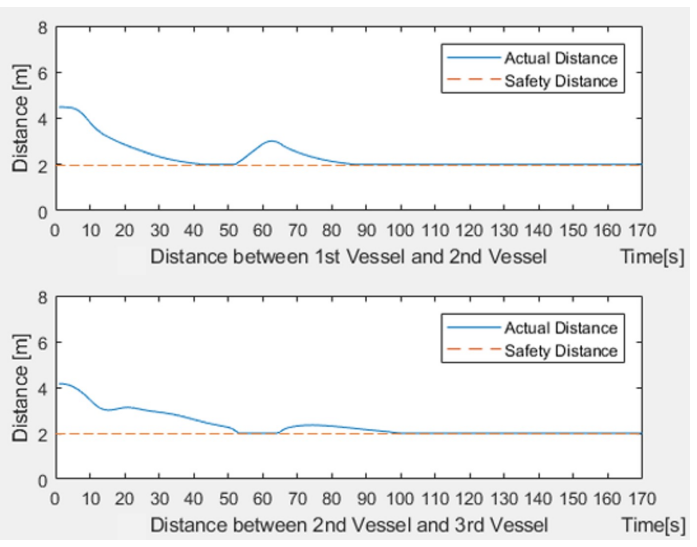

(c)

Figure 8. Unmanned vessel using constant safety distance: (a) linear velocity of $v_{i}(k)$ in Eq. (3), (b) angular velocity $w_{i}(k)$ in Eq. (3), and (c) safety distance calculated as $S_{d i}(k)=2$ in Eq. (4).

\section{Conclusions}

In this paper, a platoon of vessels longitudinal control method using a V-REP simulator is presented; proposing an effective WWV.ijfis.org

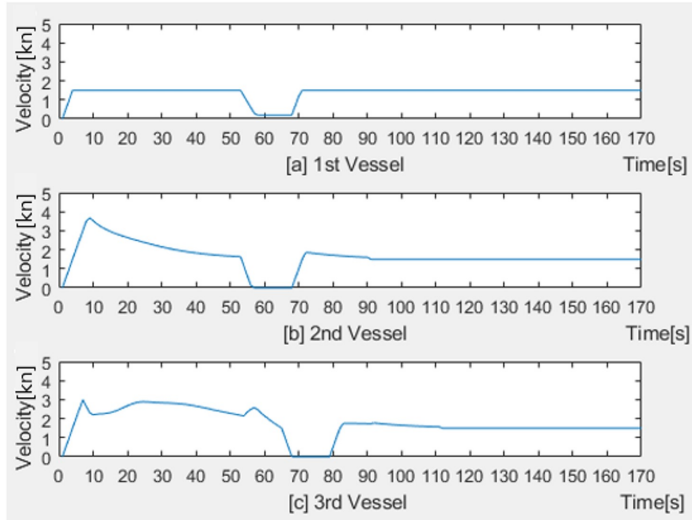

(a)

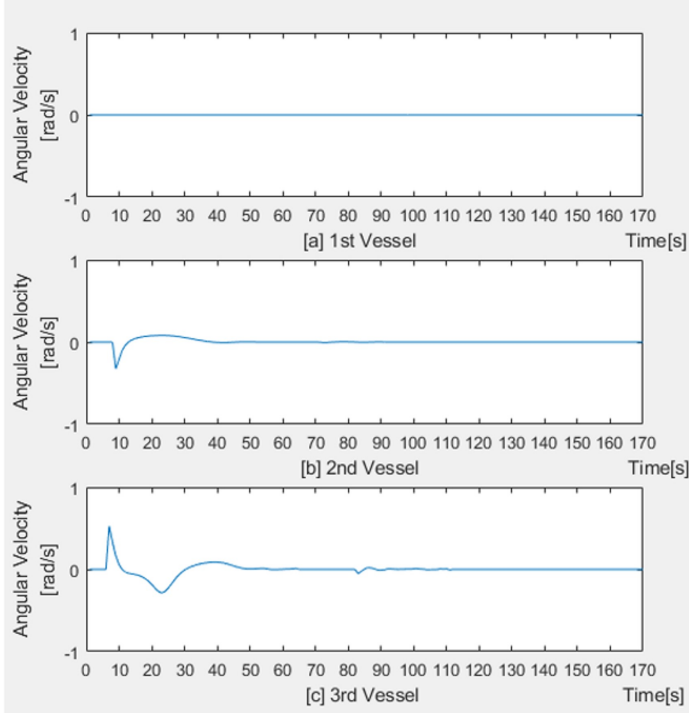

(b)

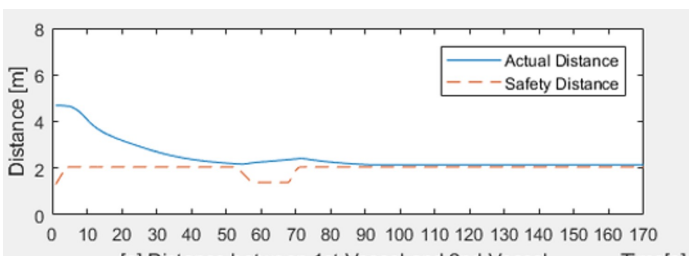

[a] Distance between 1st Vessel and 2nd Vessel Time[s]

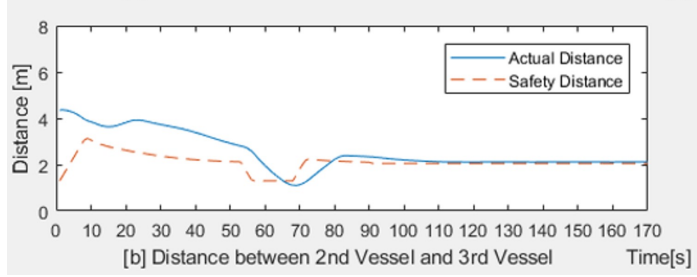

(c)

Figure 9. Unmanned vessel using the proposed method: (a) linear velocity of $v_{i}(k)$ in Eq. (3), (b) angular velocity $w_{i}(k)$ in Eq. (3), and (c) safety distance calculated as $S_{d i}(k)=\rho v_{i}(k)+L$ in Eq. (4). 


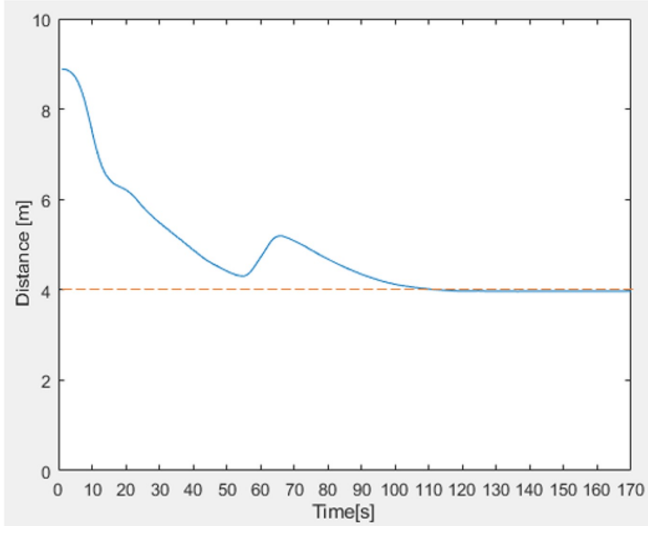

(a)

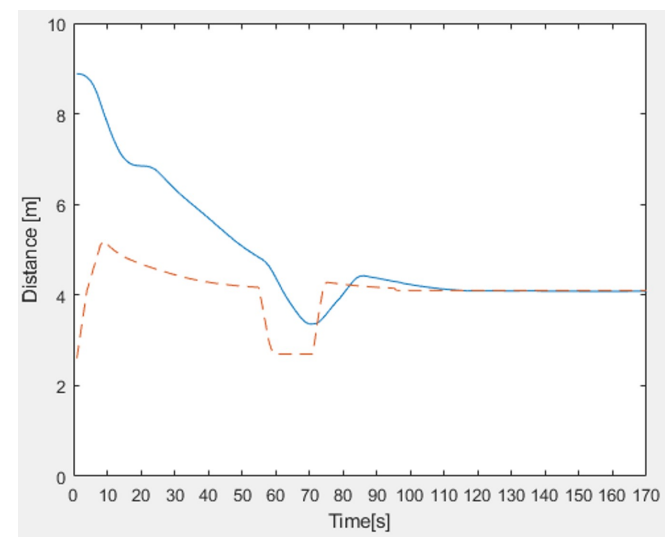

(b)

Figure 10. Total safe distance between vessels in Eq. (11): unmanned vessel using (a) constant safety distance and (b) the proposed method.

platoon of vessels scheme in the V-REP environment; using a variable safe distance between preceding and trailing vessels based on the California rule. In the conventional California rule platoon scheme, the preceding vessel moves without considering its trailing vessel position. For that reason, a compact formation among vessels is not achieved. The proposed method satisfies compact formation among vessels even when another vessel cuts across the platoon. Since the preceding vessel considers its trailing vessel speed, the distance between the preceding and trailing vessels is maintained without a large gap between two vessels. Thus, the proposed method is more appropriate for variable distance maintenance than constant distance, or even using the California rule alone.

\section{Conflict of Interest}

No potential conflict of interest relevant to this article was reported.

\section{Acknowledgements}

This research was supported by the Basic Science Research Program through the National Research Foundation of Korea (NRF) funded by the Ministry of Education, Science and Technology (NRF-2017R1A2B4011329).

\section{References}

[1] S. Garg, R. K. Singh, and R. Kapoor, "Autonomous ship navigation system," in Proceedings of 2013 Texas Instruments India Educators' Conference, Bangalore, India, 2013, pp. 300-305. http://10.1109/TIIEC.2013.60

[2] W. C. Tan, C. Y. Weng, Y. Zhou, K. H. Chua, and I. M. Chen, "Historical data is useful for navigation planning: data driven route generation for autonomous ship," in Proceedings of 2018 IEEE International Conference on Robotics and Automation (ICRA), Brisbane, Australia, 2018, pp. 7478-7483. http://10.1109/ICRA.2018.8460880

[3] J. Ning, J. Sun, T. Li, and W. Qiao, "Ship's track keeping based on variable structure active-disturbance rejection control," in Proceedings of 2015 6th International Conference on Intelligent Control and Information Processing (ICICIP), Wuhan, China, 2015, pp. 421-425. http://10.1109/ICICIP.2015.7388208

[4] Y. Lin, X. Chen, and T. Zhou, "Nonlinear PID design for ship course autopilot control," Marine Electric \& Electronic Engineering, vol. 29, no. 6, pp. 46-49, 2009.

[5] K. Y. Pettersen and E. Lefeber, "Way-point tracking control of ships," in Proceedings of the 40th IEEE Conference on Decision and Control (Cat. No. O1CH37228), Orlando, FL, 2001, pp. 940-945. http://10.1109/CDC.2001.980230

[6] J. Da, Y. Yang, G. Li, and W. Li, "Robust adaptive nonlinear controller design for dynamic positioning system of ships," in Proceedings of the 31 st Chinese Control Conference, Hefei, China, 2012, pp. 904-909.

[7] R. Smierzchalski, "The structure of the control system for a dynamically positioned ship," in Proceedings of 2016 21 st International Conference on Methods and Models in Automation and Robotics (MMAR), Miedzyzdroje, Poland, 2016, pp. 641-644. http://10.1109/MMAR.2016.7575211

[8] A. Witkowska, "Allocation methods of control signals in the dynamic positioning system of ship: current problems 
of automation and robotics," in EXIT. Warszawa, Poland: 2014, pp. 634-643.

[9] Cavotech, "Automated Mooring Systems," 2004; Available http://www.cavotec.com.ua/download/cat9/AMS.pdf

[10] C. Bergenhem, S. Shladover, E. Coelingh, C. Englund, and S. Tsugawa, "Overview of platooning systems," in Proceedings of the 19th ITS World Congress, Vienna, Austria, 2012.

[11] S. Zhao, T. Zhang, N. Wu, H. Ogai, and S. Tateno, "Vehicle to vehicle communication and platooning for EV with wireless sensor network," in Proceedings of 2015 54th Annual Conference of the Society of Instrument and Control Engineers of Japan (SICE), Hangzhou, China, 2015, pp. 1435-1440. http://10.1109/SICE.2015.7285493

[12] A. P. Colling and R. G., Hekkenberg, "A multi-scenario simulation transport model to assess the economics of semi-autonomous platooning concepts," in Proceedings of International Conference on Computer Applications and Information Technology in the Maritime Industries (COMPIT), Tullamore, Ireland, 2019, pp. 132-145.

[13] S. Azak and E. Erdogan, "Performance evaluation of the grid-based FastSLAM in V-REP using MATLAB," in Proceedings of the 14th International Conference on Advanced Trends in Radioelecrtronics, Telecommunications and Computer Engineering (TCSET), Slavske, Ukraine, 2018, pp. 276-281. IEEE.

[14] V-REP Virtual Robot Experimentation Platform, Available http://www.coppeliarobotics.com/

[15] X. Li, G. Jia, S. Wen, J. Feng, and J. Song, "Research on the control strategy of unmanned tracking ship based on GPS," in Proceedings of the 31st Chinese Control Conference, Hefei, China, 2012, pp. 4445-4449.

[16] Y. Peng, D. Qu, Y. Zhong, S. Xie, J. Luo, and J. Gu, "The obstacle detection and obstacle avoidance algorithm based on 2-D LiDAR," in Proceedings of 2015 IEEE International Conference on Information and Automation, Lijiang, China, 2015, pp. 1648-1653. http://10.1109/ICInfA. 2015.7279550

[17] D. Ghorpade, A. D. Thakare, and S. Doiphode, "Obstacle detection and avoidance algorithm for autonomous mobile robot using 2D LiDAR," in Proceedings of 2017
International Conference on Computing, Communication, Control and Automation (ICCUBEA), Pune, India, 2017, pp. 1-6. http://10.1109/ICCUBEA.2017.8463846

[18] Y. G. Kim, S. H. Lee, and D. H. Kim, "User following mobile robot system based on GPS and ultrasonic sensors," Communication in Computer and Information Science, vol. 206, pp. 295-302, Sept. 2011. https://doi.org/10.1007/ 978-3-642-24106-2_39

[19] W. Naeem, T. Xu, R. Sutton, and A. Tiano, "The design of a navigation, guidance, and control system for an unmanned surface vehicle for environmental monitoring," Proceedings of the Institution of Mechanical Engineers, Part M: Journal of Engineering for the Maritime Environment, vol. 222, no. 2, pp. 67-79, 2008. https://doi.org/10.1243/14750902JEME80

[20] L. Qiao and Y. Yang, "Design of PD observer-based faulttolerant controller for descriptor systems: application to a ship propulsion system," in Proceedings of the 37th Chinese Control Conference (CCC), Wuhan, China, 2018, pp. 6033-6037. http://10.23919/ChiCC.2018.8482842

[21] Z. Li, J. Hu, and X. Huo, "PID control based on RBF neural network for ship steering," in Proceedings of 2012 World Congress on Information and Communication Technologies, Trivandrum, India, 2012, pp. 1076-1080. http://10.1109/WICT.2012.6409235

[22] C. C. Chien and P. Ioannou, "Automatic vehiclefollowing," in Proceedings of 1992 American Control Conference, Chicago, IL, 1992, pp. 1748-1752. http: //10.23919/ACC.1992.4792410

[23] Y. Melhaoui, A. A. Allal, A. Kamil, K. Mansouri, and M. Rachik, "Toward an overview of ship collision avoidance maneuvers approaches in compliance with COLREG convention," in Proceedings of the 5th International Conference on Optimization and Applications (ICOA), Kenitra, Morocco, 2019, pp. 1-4. http://10.1109/ICOA.2019. 8727687

[24] Y. Kuwata, M. T. Wolf, D. Zarzhitsky, and T. L. Huntsberger, "Safe maritime autonomous navigation with COLREGS, using velocity obstacles," IEEE Journal of Oceanic Engineering, vol. 39, no. 1, pp. 110-119, 2013. http://10.1109/JOE.2013.2254214 


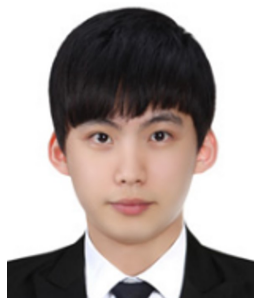

Myoung-Sung Kim received his B.S. degree from the Department of Electrical Engineering, Kyungnam University, Korea, in 2017. He is currently a M.S. candidate in the Department of Mechatronics Engineering, Kyungnam University. His research interests include platoon of vessels, mobile robots, and behavior algorithm.

E-mail: muldonge3@naver.com

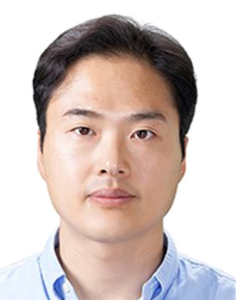

Kwang-Kook Lee received his degrees from the Department of Naval Architecture \& Ocean Engineering, Seoul National University, Korea, in 2008. From 2008 to 2011, he was a manager of production planning team in STX Offshore \& Shipbuilding. From 2019 to 2020, he joined University of Southampton, UK, as a visiting professor. Since 2011, he has been an associate professor at the Department of Naval Architecture \& Ocean System Engineering, Kyungnam University, Korea. His research interests include marine system design and control, modelling and simulation, and hardware-in-the-loop simulation.

E-mail: kklee@kyungnam.ac.kr

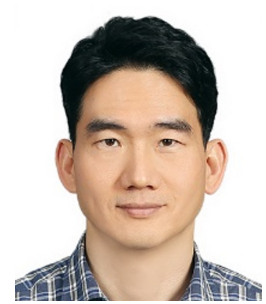

Dong Hun Kim received his B.S., M.S., and Ph.D. degrees from the Department of Electrical Engineering, Hanyang University, Korea, in 1995, 1997, and 2001, respectively. From 2001 to 2003, he was a research associate under several grants in the Department of Electrical and Computer Engineering, Duke University, NC, USA. In 2003, he joined Boston University, MA, USA, as a visiting assistant professor under several grants at the Department of Aerospace and Mechanical Engineering. In 2004, he was engaged in post-doctoral research at the School of Information Science and Technology, the University of Tokyo, Japan. Since 2005, he has been a professor at the Department of Electrical Engineering, Kyungnam University, Korea. His research interests include swarm robotics, mobile robots, decentralized control of autonomous vehicles, intelligent control, and adaptive nonlinear control.

E-mail: dhkim@kyungnam.ac.kr 